Methods: Two hundred and fifty-five patients fulfilling the imaging arm of the Assessment of SpondyloArthritis International Society axSpA criteria were enrolled. TBS and bone mineral density (BMD) were assessed using dual-energy X-ray absorptiometry. Vertebral fracture of the thoracic and lumbar spine was defined according to the Genant criteria. Osteoporosis risk factors, inflammatory markers, disease activity scores, and spinal structural damage were also assessed. Multivariate logistic regression analysis was performed to identify factors associated with vertebral fractures.

Results: Of 255 axSpA patients, 28 (11\%) had 31 vertebral fractures. The mean TBS was $1.39 \pm 0.11$ and $1.30 \pm 0.13$ in patients without and with vertebral fractures, respectively $(<0.001)$. BMD in the femoral neck was lower in patients with vertebral fractures $(p=0.027)$, but BMDs in the lumbar spine and total hip were not. In the multivariate analyses, low TBS and the presence of syndesmophytes were significantly associated with vertebral fractures, independently of BMD (OR $[95 \% \mathrm{Cl}]=3.6[1.2-11.1]$ and $3.3[1.0-10.7]$, respectively). For the total hip, TBS has a better discriminatory value than BMD for prediction of vertebral fractures in axSpA patients $(\mathrm{p}=0.034)$.

Conclusions: TBS values are lower in axSpA patients with vertebral fractures. Low TBS and syndesmophytes were independently associated with prevalent vertebral fractures. TBS has better predictive value to BMD for the discrimination of vertebral fractures and could help to detect axSpA patients with vertebral fractures.

Disclosure of Interest: None declared

DOI: 10.1136/annrheumdis-2018-eular.1712

\section{THU0258 DIAGNOSTIC VALUE OF ANTI-CD74 AUTOANTIBODIES IN AXIAL SPONDYLOARTHRITIS AND AXIAL PSORIATIC ARTHRITIS. RESULTS OF OPEN-LABEL, CROSS- SECTIONAL, CONTROLLED, MULTICENTER PROGRESS STUDY}

1.Z. Gaydukova ${ }^{1}$, A. Rebrov ${ }^{2}$, A. Maslyanskiy $^{3}$, S. Lapin ${ }^{4}$, E. Vasilenko ${ }^{5}$, V. Mazurov ${ }^{5}$. ${ }^{1}$ North-Western State Medical University n.a.ll Mechnicov, St Petersburg, Russian Federation; ${ }^{2}$ Saratov State Medical University, Saratov, ${ }^{3}$ V.A. Almazov North-Western Federal Medical Research Centre; ${ }^{4}$ First Saint Petersburg State Medical University named after I.P. Pavlov, ${ }^{5}$ North-Western State Medical University, St Petersburg

Background: The problem of axial spondyloarthritis' (axSpA) differential diagnostics is not solved, especially in the early stages of the axSpA. Therefore, new diagnostic markers for axSpA are needed.

Objectives: of the study were to evaluate the prevalence, sensitivity and specificity of anti-CD74 autoantibodies (anti-CD74-AB) in HLA-B27 associated axSpA in comparison with HLA-B27 associated axial Psoriatic arthritis (axPsA) and with healthy controls.

Methods: Anti-CD74-AB (quantitative ELISA) were measured in serum of 114 HLA-B27-positive patients with axSpA, and in 26 age- and sex - matched HLAB27 positive patients with axPsA, and in 37 healthy controls without HLA-B27. 68 axSpA patients had ankylosing spondylitis (AS) according mNew-York criteria (1984), 46 axSpA persons had non-radiographic axSpA (nr-axSpA) due to ASAS criteria for axSpA (2009). AxPsA patients had psoriatic arthritis with axial involvement and fulfilled both CASPAR (2006) and ASAS axSpA criteria (2009). Disease activity in axSpA and axPsA patients was measured according ASAS recommendations. ${ }^{1}$

Results: Patients with AS, nr-axSpA and axPsA were comparable in SpA activity: differences in BASDAI, ASDAS indices and C-reactive protein levels were not significant, $\mathrm{p}>0.05$ for all. $\mathrm{Nr}$-axSpA patients had shorter disease duration as compared with $A S$ and axPsA patients $(p<0.001)$.

The concentration of anti-CD74-AB in patients with axSpA was $3.5 \pm 3.0 \mathrm{U} / \mathrm{ml}(3.1$ $\pm 3.0 \mathrm{U} / \mathrm{ml}$ in $\mathrm{AS}$ and $3.8 \pm 2.9 \mathrm{U} / \mathrm{ml}$ in $\mathrm{nr}$-axSpA patients), $2.1 \pm 1.4 \mathrm{U} / \mathrm{ml}$ in patients with axPsA ( $p \geq 0.05$ compared to controls and ax-SpA)) and $1.3 \pm 1.4 \mathrm{U} / \mathrm{ml}$ in healthy controls ( $p<0.05$ for the difference with ax-SpA, AS and nr-axSpA). Diagnostic values of anti-CD74-AB in axSpA (ROC-analyse results) are presented in table 1.

Abstract THU0258 - Table 1. Diagnostic values of anti-CD74 autoantibodies in patients with axial spondyloarthritis

(ROC-analyse):

\begin{tabular}{lcccccc}
\hline & $\begin{array}{c}\mathrm{AUC} \\
(95 \% \mathrm{Cl})\end{array}$ & $\begin{array}{c}\text { Sensitivity } \\
\text { of the test, } \\
\%\end{array}$ & $\begin{array}{c}\text { Specificity } \\
\text { of the test, } \\
\%\end{array}$ & $+\mathrm{LR}$ & $\begin{array}{c}\text { Upper cut-off value for } \\
\text { reference interval, U/ml }\end{array}$ & $\mathrm{p}$ \\
\hline axSpA & $\begin{array}{c}0,74 \\
(0,67-\end{array}$ & 64,4 & 89,2 & 5,9 & $>2,0$ & $<0,0001$ \\
& $\begin{array}{c}0,82) \\
\text { AS }\end{array}$ & & & & & 0001 \\
& $\begin{array}{c}0,68 \\
(0,59- \\
0,79)\end{array}$ & 60,3 & 89,2 & 5,6 & $>2,0$ & \\
& & & & & &
\end{tabular}

\begin{tabular}{lcccccc} 
nr- & 0,78 & 73,1 & 84,0 & 4,5 & $>1,73$ & $<0,0001$ \\
$\operatorname{axSpA}$ & $\begin{array}{c}0,58- \\
0,83)\end{array}$ & & & & & \\
axPsA & $\begin{array}{c}0,7(0,69- \\
0,89)\end{array}$ & 96,1 & 45,9 & 1,8 & 0,7 & 0005 \\
\hline
\end{tabular}

AS - ankylosing spondylitis, axSpa - axial spondyloarthritis, axPsA - axial psoriatic arthritis, nr-axSpA - non-radiographic axial spondyloarthritis, $\mathrm{Cl}$ - confidence interval, $+L R-$ positive likelihood ratio.

Cut-off value of anti-CD74-AB>2.0 U/ml in patients with axSpA showed the diagnostic sensitivity of $64.4 \%$, specificity of $89.2 \%$, the positive LR of 5.9 , whereas in patients with nr-axSpA at concentrations of $1.7 \mathrm{U} / \mathrm{ml}$ - sensitivity $73.1 \%$, specificity $84 \%$ and positive LR 4.5 .

Conclusions: Conclusions. Anti-CD74-AB are strongly associated with nraxSpA and less - axPsA. The measurement of anti-CD74-AB can be considered as candidate biomarker in the diagnostics of axSpA and in differential diagnostics between HLA-B27 positive ax-SpA and axPsA, especially in early stages of the diseases. Further studies are needed for the evaluation of anti-CD74-AB diagnostic capacity.

\section{REFERENCE:}

[1] Sieper J, et al. Ann Rheum Dis 2009 Jun;68 Suppl 2:ii1-44.

Disclosure of Interest: None declared

DOI: 10.1136/annrheumdis-2018-eular.4892

\section{THU0259 FREQUENCY AND PATTERN OF THE UVEITIS IN SPONDYLOARTHRITIS WITH BIOLOGICAL THERAPY}

$\underline{\text { I. Calvo }}^{1}$, E. Guerrero ${ }^{1}$, O. Ibarguengoitia ${ }^{1}$, D. Montero ${ }^{1}$, M.L. Garcia ${ }^{1}$, E. Ruiz ${ }^{1}$, I. Torre ${ }^{1}$, O. Fernandez ${ }^{1}$, J.M. Blanco ${ }^{1}$, A.R. Intxaurbe ${ }^{1}$, C. Perez ${ }^{1}$, I. Gorostiza ${ }^{2}$, E. Galindez ${ }^{1}{ }^{1}$ Rheumatology; ${ }^{2}$ Research Unit, Basurto University Hospital, BILBAO, Spain

Background: Uveitis is the most frequent extra-articular manifestation (EAM) of spondyloarthritis (SpA). Its prevalence is approximately $30 \%$ and increases with the duration of the $\mathrm{SpA}$. The characteristic pattern is anterior, acute, recurrent and unilateral uveitis. However, the frequency and characteristics of uveitis in SpA treated with biological therapy (BT) are unknown.

Objectives: The main target is to describe the frequency and characteristics of uveitis in SpA with BT in a single centre.

Methods: Descriptive and retrospective study (January 2003-December 2017) of $\mathrm{SpA}$ that develops uveitis in a reference hospital. The epidemiological variables, type of SpA, presence of uveitis and its characteristics, presence of BT at the time of onset and treatment received are collected. For the analysis, frequencies and percentages were used in qualitative variables, and mean and standard deviation (SD) for quantitative variables. Statistical analysis was performed with IBM SPSS v.23.

Results: We studied 246 patients with SpA. The subtypes of SpA were: ankylosing spondylitis (AS) $(n=125,50.8 \%)$, psoriatic arthritis (PsA) $(n=101,41.1 \%)$, undifferentiated $\operatorname{SpA}(n=13,5.3 \%)$, non-radiographic axial Spa $(n=3,1.2 \%)$, enteropathic arthropathy $(n=3,1.2 \%)$ and reactive arthritis $(n=1,0.4 \%)$.

Uveitis was observed in 41 patients (16.7\%) after an average time of development of 109.47 (73.9) months of the SpA. The incidence rate was 5.5 cases of uveitis/ 100 patients-year of follow-up. $70.7 \%$ were men and the mean age(SD) was 47.4 (12.06) years. $87.8 \%$ of the cases were HLAB27 positive and had a family history of $\mathrm{SpA} 41.5 \%$.

Uveitis was observed in 33 patients $(80.5 \%)$ with AS, in 6 (14.6\%) with PsA, in 1 $(2.4 \%)$ with non-Rx axial SpA and in $1(2.4 \%)$ with undifferentiated SpA. (table 1 ) The uveitis pattern was anterior (100\%), acute (92.7\%), unilateral $(87.8 \%)$ and in $12.2 \%$ bilateral $(80 \%$ in PsA). At the time of onset of uveitis, the mean ESR was $30.11 \mathrm{~mm}^{\text {ah }}$, CRP $3.56 \mathrm{mg} / \mathrm{dL}$, DAS28 3.66 and BASDAI 3.21 .

Regarding the diagnosis of SpA, uveitis was after $(85.4 \%)$, before $(12.2 \%)$ and simultaneous (2.4\%).

At the time of the onset of uveitis, 14 patients $(34.1 \%)$ were with BT (35.7\% etanercept, $28.6 \%$ infliximab, $21.4 \%$ adalimumab, $7.1 \%$ golimumab and $7.1 \%$ certolizumab). BT was modified in 3 of the cases.

The treatment of uveitis was topical $(78 \%)$, corticoids in oral regimen $(57.5 \%)$ conventional DMARDs (12.5\%), with methotrexate predominating in $60 \%$ of cases and BT (15\%). The most used biologics were adalimumab $(50 \%)$, infliximab (33.3\%) and sekukinumab (16.7\%). 